\title{
Energy and Momentum in Spacetime Homogeneous Gödel-type Metrics
}

\author{
M. Sharif* \\ Department of Mathematics, University of the Punjab, \\ Quaid-e-Azam Campus Lahore-54590, PAKISTAN, \\ $<$ hasharif@yahoo.com>
}

\begin{abstract}
Using Einstein and Papapetrou energy-momentum complexes, we explicitly calculate the energy and momentum distribution associated with spacetime homogeneous Gödel-type metrics. We obtain that the two definitions of energy-momentum complexes do not provide the same result for these type of metrics. However, it is shown that the results obtained are reduced to the energy-momentum densities of Gödel metric already available in the literature.
\end{abstract}

Key Words: Energy and Momentum, Gödel-type Metrics PACS: 04.20.Cv

*Present Address: Department of Mathematical Sciences, University of Aberdeen, Kings College, Aberdeen AB24 3UE Scotland, UK. <msharif@maths.abdn.ac.uk> 


\section{Introduction}

The problem of energy and momentum has been one of the oldest but most interesting problems in Einstein's theory of General Relativity (GR). Due to its peculiar nature and diverse points of view, it has been the most controversial problem. In a curved spacetime the energy-momentum tensor of matter plus all non-gravitational fields no longer satisfies $T_{a ; b}^{b}=0$. The contribution from the gravitational field is now required to construct an energymomentum expression which satisfies a divergence relation. Einstein himself started work to solve this problem and suggested an expression for energymomentum distribution [1]. He justified that his energy-momentum complex provides convincing results for the total energy and momentum of isolated systems. After this, many physicists including Landau-Lifshitz [2], Tolman [3], Papapetrou [4], Bergmann [5], Weinberg [6] had suggested different expressions for the energy-momentum distribution. The main problem with these definitions is that they are coordinate dependent. One can have meaningful results only when calculations are performed in Cartesian coordinates. This restriction of coordinate dependent motivated some other physicists like Möller [7]-[8], Komar [9] and Penrose [10] who constructed coordinate independent definitions of energy-momentum complex.

Möller claimed that his expression gives the same values for the total energy and momentum as the Einstein's energy-momentum complex for a closed system. However, Möller's energy-momentum complex was subjected to some criticism [8]-[11]. Komar's prescription, though not restricted to the use of Cartesian coordinates, is not applicable to non-static space-times. Penrose [10] pointed out that quasi-local masses are conceptually very important. The inadequacies of these quasi-local masses (these different definitions do not give agreed results for the Reissner- Nordstrom and Kerr metrics and that the Penrose definition could not succeed to deal with the Kerr metric) have been discussed in [12]-[14]. Thus each of these energy-momentum complex has its own drawback. As a result these ideas of the energy-momentum complex were severally criticized.

Virbhadra [14]-[15] was the first who revived the interest in this approach. Since then lot of work on evaluating the energy-momentum distributions of different spacetimes have been carried out by different authors [16]-[19]. In a recent paper, Virbhadhra [14] investigated whether or not the energymomentum complexes of Einstein, Landau and Lifshitz, Papapetrou and Weinberg give the same energy distribution for the most general non-static 
spherically symmetric metric. It was a great surprise that contrary to previous results of many asymptotically flat spacetimes and asymptotically nonflat spacetimes, he found that these definitions disagree. He observed that Einstein's energy-momentum complex provides a consistent result for the Schwarzschild metric whether one calculates in Kerr-Schild Cartesian coordinates or Schwarzschild Cartesian coordinates. The prescriptions of LandauLifshitz, Papapetrou and Weinberg furnish the same result as in the Einstein prescription if the calculations are carried out in Schwarzschild Cartesian coordinates. Thus the prescriptions of Landau-Lifshitz, Papapetrou and Weinberg do not give a consistent result. On the basis of these and some other facts $[12,13]$, Virbhadra concluded that the Einstein method seems to be the best among all known (including quasi-local mass definitions) for energy distribution in a spacetime. Recently, Lessner [20] pointed out that the Möller's energy-momentum prescription is a powerful concept of energy and momentum in GR.

In a series of papers [21] Cooperstock has propounded a hypothesis according to which, in a curved spacetime, energy and momentum are confined to the regions of non-vanishing energy-momentum tensor $T_{a}^{b}$ of the matter and all non-gravitational fields. The results of Xulu [22] and the recent results of Bringley [23] support this hypothesis. It would be interesting to investigate further whether or not the Cooperstock's hypothesis stands true. In a recent paper [19], we have applied Einstein and Papapetrou's prescriptions to calculate energy-momentum densities of Gödel spacetime. The results obtained for the Gödel metric are much simple in both the prescriptions. We obtain that the energy density is exactly the same in both the prescriptions with the exception of different signs in the first term while the momentum density components exactly coincide up to the first term only.

In this paper we extend the procedure and use Einstein and Papapetrou's prescriptions to evaluate energy and momentum densities in Gödel-type metrics. As we shall see from the analysis given in the paper, when procedure is extended to such metrics, the problem becomes considerably complicated. We find that the results obtained by these two prescriptions are not the same. However, it is shown that they both reduce to the known results for particular values of $H$ and $D$, i.e., Gödel spacetime. In the next section, we shall describe the spacetime homogeneous Gödel-type metrics. In sections three and four, we evaluate energy and momentum using Einstein and Papapetrou's prescriptions respectively. Finally, the results obtained will be concluded. 


\section{Spacetime Homogeneous Gödel-type Met- rics}

A solution of Einstein's field equations with cosmological constant for incoherent matter with rotation was found by Gödel. This is the best known example of a cosmological model which makes it apparent that GR does not exclude the existence of a closed timelike world-lines, despite its Lorentzian character which leads to the local validity of the causality principle. Gödeltype metrics are given by the line element of the form [24]

$$
d s^{2}=[d t+H(r) d \theta]^{2}-d r^{2}-D^{2}(r) d \theta^{2}-d z^{2},
$$

in the cylindrical coordinates $(r, \theta, z)$. Here the metric functions $H$ and $D$ depend on the coordinate $r$ only. It admits a five-parameter group of isometries $\left(G_{5}\right)$ having an isotropy subgroup of dimension one $\left(H_{1}\right)$.

Raychaudhuri and Thakurta [25] are the first who have determined the necessary conditions for a Gödel-type metric to be a spacetime homogeneous (hereafter called ST homogeneous). Later, Reboucas and Tiomno [26] proved that these conditions are also sufficient for ST homogeneity of Gödel-type Riemannian spacetime manifolds. These necessary and sufficient conditions are given by

$$
\begin{aligned}
& \frac{D^{\prime \prime}}{D}=\text { constant } \equiv m^{2}, \\
& \frac{H^{\prime}}{D}=\text { constant } \equiv-2 \omega .
\end{aligned}
$$

The necessary and sufficient conditions were finally re-derived for a Gödeltype manifold to be ST homogeneous without assuming any such simplifying hypothesis in [27].

We can distinguish the ST metrics in the following four classes as given in [28-29] according to

Class I: $m^{2}>0, \omega \neq 0$. In this case, the general solution of Eqs.(2) and (3) is given by

$$
H(r)=\frac{2 \omega}{m^{2}}[1-\cosh (m r)], \quad D(r)=\frac{1}{m} \sinh (m r) .
$$

Class II: $m^{2}=0, \omega \neq 0$. For this class, the general solution of Eqs.(2) and (3) can be written as

$$
H(r)=-\omega r^{2}, \quad D(r)=r .
$$


Class III: $m^{2} \equiv-\mu^{2}, \omega \neq 0$. If we integrate Eqs.(2) and (3) for this case, we have the following solution

$$
H(r)=\frac{2 \omega}{\mu^{2}}[\cos (\mu r)-1], \quad D(r)=\frac{1}{\mu} \sin (\mu r) .
$$

Class IV: $m^{2} \neq 0, \omega=0$. In this case, the cross term related to the rotation $\omega$ in the Gödel model vanishes. Consequently, one can make $H=0$ by a trivial coordinate transformation.

If $m^{2}=0=\omega$, the line element (1) becomes Minkowskian. Also, it is mentioned that the case $m^{2}=2 \omega^{2}$ defines the original Gödel metric.

In order to have meaningful results in the prescriptions of Einstein and Papapetrou, it is necessary to transform the metric in Cartesian coordinates. We transform the metric in Cartesian coordinates by using

$$
x=r \cos \theta, \quad y=r \sin \theta .
$$

The corresponding metric in these coordinates will become

$d s^{2}=d t^{2}-\frac{1}{r^{2}}(x d x+y d y)^{2}+\frac{1}{r^{4}}\left(H^{2}-D^{2}\right)(x d y-y d x)^{2}-d z^{2}+\frac{2}{r^{2}} H d t(x d y-y d x)$.

\section{Energy and Momentum in Einstein's Pre- scription}

The energy-momentum complex of Einstein [1] is given by

$$
\Theta_{a}^{b}=\frac{1}{16 \pi} M_{a, c}^{b c}
$$

where

$$
M_{a}^{b c}=\frac{g_{a d}}{\sqrt{-g}}\left[-g\left(g^{b d} g^{c e}-g^{c d} g^{b e}\right)\right]_{, e}, \quad a, b, c, d, e=0,1,2,3
$$

$\Theta_{0}^{0}$ is the energy density, $\Theta_{0}^{a}$ are the momentum density components, and $\Theta_{a}^{0}$ are the components of energy current density. The Einstein energymomentum satisfies the local conservation laws

$$
\frac{\partial \Theta_{a}^{b}}{\partial x^{b}}=0 .
$$


In order to evaluate the energy and momentum densities in Einstein's prescription associated with Gödel-type metrics, we need to calculate the nonvanishing components of $M_{a}^{b c}$

$$
\begin{gathered}
M_{0}^{01}=\frac{1}{D r^{3}}\left(D^{2} x+H H_{1} x^{2}+H H_{2} x y-2 D D_{1} x^{2}-2 D D_{2} x y+r^{2} x\right) \\
M_{1}^{01}=-\frac{1}{D r^{5}}\left(H^{2} H_{1} x^{2} y+H^{2} H_{2} x y^{2}-2 H D D_{1} x^{2} y-2 H D D_{2} x y^{2}\right. \\
\left.-H_{1} r^{2} x^{2} y+H_{2} r^{2} x^{3}+D^{2} H_{1} x^{2} y+D^{2} H_{2} x y^{2}\right)
\end{gathered}
$$

It is to be noted that $H_{1}$ and $H_{2}$ denote differentiation of $H$ with respect to the coordinates $x$ and $y$ respectively. Using Eqs.(12)-(18) in Eq.(9), we obtain the energy and momentum densities in Einstein's prescription

$$
\begin{array}{r}
\Theta_{0}^{0}=\frac{1}{16 \pi D^{2} r^{3}}\left(-D^{3}+D^{2} D_{1} x+D^{2} D_{2} y-2 D^{2} D_{11} x^{2}-2 D^{2} D_{22} y^{2}\right. \\
-4 D^{2} D_{12} x y+D r^{2}-D_{1} r^{2} x-D_{2} r^{2} y+H H_{11} D x^{2}+H H_{22} D y^{2} \\
+2 H H_{12} D x y+2 H_{1} H_{2} D x y+H_{1}^{2} D x^{2}+H_{2}^{2} D y^{2} \\
\left.-H H_{1} D_{1} x^{2}-H H_{2} D_{1} x y-H H_{1} D_{2} x y-H H_{2} D_{2} y^{2}\right)
\end{array}
$$




$$
\begin{aligned}
& \Theta_{1}^{0}=\frac{1}{16 \pi D^{2} r^{5}}\left(H^{2} H_{1} D x y+H^{2} H_{2} D y^{2}-H^{2} H_{11} D x^{2} y-H^{2} H_{22} D y^{3}\right. \\
& -2 H^{2} H_{12} D x y^{2}+H^{2} H_{1} D_{1} x^{2} y+H^{2} H_{1} D_{2} x y^{2}+H^{2} H_{2} D_{1} x y^{2} \\
& +H^{2} H_{2} D_{2} y^{3}-2 H H_{1}^{2} D x^{2} y-2 H H_{2}^{2} D y^{3}-4 H H_{1} H_{2} D x y^{2} \\
& -2 H D^{2} D_{1} x y-2 H D^{2} D_{2} y^{2}+2 H D^{2} D_{11} x^{2} y+2 H D^{2} D_{22} y^{3} \\
& +4 H D^{2} D_{12} x y^{2}+D^{3} H_{1} x y+D^{3} H_{2} y^{2}-D^{3} H_{11} x^{2} y-D^{3} H_{22} y^{3} \\
& -2 D^{3} H_{12} x y^{2}+D^{2} D_{1} H_{1} x^{2} y+D^{2} D_{1} H_{2} x y^{2}+D^{2} D_{2} H_{1} x y^{2} \\
& +D^{2} D_{2} H_{2} y^{3}+H D r^{2} y+H D_{2} r^{4}+D H_{1} r^{2} x y-D H_{2} r^{2} x^{2} \\
& -D H_{2} r^{4}+D H_{11} r^{2} x^{2} y-D H_{22} r^{2} x^{2} y+D H_{12} r^{2} x y^{2} \\
& \left.-D H_{12} r^{2} x^{3}-H_{1} D_{1} r^{2} x^{2} y-H_{1} D_{2} r^{2} x y^{2}+H_{2} D_{1} r^{2} x^{3}+H_{2} D_{2} r^{2} x^{2} y\right) \text {, } \\
& \Theta_{2}^{0}=-\frac{1}{16 \pi D^{2} r^{5}}\left(H^{2} H_{2} D x y+H^{2} H_{1} D x^{2}-H^{2} H_{22} D x y^{2}-H^{2} H_{11} D x^{3}\right. \\
& -2 H^{2} H_{12} D x^{2} y+H^{2} H_{2} D_{2} x y^{2}+H^{2} H_{2} D_{1} x^{2} y+H^{2} H_{1} D_{2} x^{2} y \\
& +H^{2} H_{1} D_{1} x^{3}-2 H H_{2}^{2} D x y^{2}-2 H H_{1}^{2} D x^{3}-4 H H_{1} H_{2} D x^{2} y \\
& -2 H D^{2} D_{2} x y-2 H D^{2} D_{1} x^{2}+2 H D^{2} D_{22} x y^{2}+2 H D^{2} D_{11} x^{3} \\
& +4 H D^{2} D_{12} x^{2} y+D^{3} H_{2} x y+D^{3} H_{1} x^{2}-D^{3} H_{22} x y^{2}-D^{3} H_{11} x^{3} \\
& -2 D^{3} H_{12} x^{2} y+D^{2} D_{2} H_{2} x y^{2}+D^{2} D_{2} H_{1} x^{2} y+D^{2} D_{1} H_{2} x^{2} y \\
& +D^{2} D_{1} H_{1} x^{3}+H D r^{2} x+H D_{1} r^{4}+D H_{2} r^{2} x y-D H_{1} r^{2} y^{2} \\
& -D H_{1} r^{4}+D H_{22} r^{2} x y^{2}-D H_{11} r^{2} x y^{2}+D H_{12} r^{2} x^{2} y \\
& \left.-D H_{12} r^{2} y^{3}-H_{2} D_{2} r^{2} x y^{2}-H_{2} D_{1} r^{2} x^{2} y+H_{1} D_{2} r^{2} y^{3}+H_{1} D_{1} r^{2} x y^{2}\right) \text {, } \\
& \Theta_{0}^{1}=\frac{1}{16 \pi D^{2} r^{3}}\left(D H_{2} r^{2}-D H_{1} x y-D H_{2} y^{2}+H_{12} r^{2} x+D H_{22} r^{2} y\right. \\
& \left.-D_{2} H_{1} r^{2} x-D_{2} H_{2} r^{2} y\right) \\
& \Theta_{0}^{2}=-\Theta_{0}^{1} \text {, } \\
& \Theta_{0}^{3}=\Theta_{3}^{0}=0 .
\end{aligned}
$$

Now for $H=e^{a r}$ and $D=e^{a r} / \sqrt{2}$, Eqs.(19)-(24) become

$$
\begin{gathered}
\Theta_{0}^{0}=\frac{(1-a r)}{16 \sqrt{2} \pi r^{3}}\left[-e^{a r}+2 r^{2} e^{-a r}\right], \\
\Theta_{1}^{0}=\frac{y}{16 \sqrt{2} \pi r^{4}}\left[2 r+a(1-2 a r) e^{2 a r}\right],
\end{gathered}
$$




$$
\begin{gathered}
\Theta_{2}^{0}=-\frac{x}{16 \sqrt{2} \pi r^{4}}\left[2 r+a(1-2 a r) e^{2 a r}\right], \\
\Theta_{0}^{1}=\Theta_{0}^{2}=\Theta_{0}^{3}=\Theta_{3}^{0}=0 .
\end{gathered}
$$

These are the energy and momentum densities of Gödel spacetime given by Sharif [19].

\section{Energy and Momentum in Papapetrou's Pre- scription}

The symmetric energy-momentum complex of Papapetrou [4] is given by

$$
\Omega^{a b}=\frac{1}{16 \pi} N_{, c d}^{a b c d},
$$

where

$$
N^{a b c d}=\sqrt{-g}\left(g^{a b} \eta^{c d}-g^{a c} \eta^{b d}+g^{c d} \eta^{a b}-g^{b d} \eta^{a c}\right),
$$

and $\eta^{a b}$ is the Minkowski spacetime. The energy-momentum complex satisfies the local conservation laws

$$
\frac{\partial \Omega^{a b}}{\partial x^{b}}=0
$$

The locally conserved energy-momentum complex $\Omega^{a b}$ contains contributions from the matter, non-gravitational and gravitational fields. $\Omega^{00}$ and $\Omega^{0 a}$ are the energy and momentum (energy current) density components. To find the energy and momentum densities of the spacetime under consideration, we require the following non-zero components of $N^{a b c d}$ given as

$$
\begin{gathered}
N^{0011}=\frac{1}{D r^{3}}\left(H^{2} r^{2}-D^{2} r^{2}-D^{2} x^{2}-r^{2} y^{2}\right), \\
N^{0012}=\frac{1}{D r^{3}} x y\left(r^{2}-D^{2}\right)=N^{0021}, \\
N^{0022}=\frac{1}{D r^{3}}\left(H^{2} r^{2}-D^{2} r^{2}-D^{2} y^{2}-r^{2} x^{2}\right), \\
N^{0121}=-\frac{1}{D r} H x, \\
N^{0122}=-\frac{1}{D r} H y,
\end{gathered}
$$




$$
\begin{aligned}
& N^{0211}=-N^{0121}, \\
& N^{0212}=-N^{0122},
\end{aligned}
$$

Substituting Eqs.(32)-(38) in Eq.(29), we obtain the following energy and momentum density components in Papapetrou's prescription

$$
\begin{array}{r}
\Omega^{00}=\frac{1}{16 \pi D^{3} r^{3}}\left(H^{2} D^{2}+2 H^{2} D D_{1} x+2 H^{2} D D_{2} y+2 H^{2} D_{1}^{2} r^{2}\right. \\
+2 H^{2} D_{2}^{2} r^{2}-H^{2} D D_{11} r^{2}-H^{2} D D_{22} r^{2}-4 H H_{1} D^{2} x \\
-4 H H_{2} D^{2} y-4 H H_{1} D D_{1} r^{2}-4 H H_{2} D D_{2} r^{2}+2 H H_{11} D^{2} r^{2} \\
+2 H H_{22} D^{2} r^{2}+2 H_{1}^{2} D^{2} r^{2}+2 H_{2}^{2} D^{2} r^{2}-D^{4}+2 D^{3} D_{1} x \\
+2 D^{3} D_{2} y-D^{3} D_{11} r^{2}-D^{3} D_{22} r^{2}-D^{3} D_{11} x^{2}-D^{3} D_{22} y^{2} \\
-2 D^{3} D_{12} x y+D^{2} r^{2}-2 D D_{1} r^{2} x-2 D D_{2} r^{2} y+D D_{11} r^{2} y^{2} \\
\left.+D D_{22} r^{2} x^{2}-2 D D_{12} r^{2} x y-2 D_{1}^{2} r^{2} y^{2}-2 D_{2}^{2} r^{2} x^{2}+4 D_{1} D_{2} r^{2} x y\right), \\
\Omega^{01}=\frac{1}{16 \pi D^{3} r^{3}}\left(H D^{2} y-H D D_{1} x y+2 H D D_{2} x^{2}+H D D_{2} y^{2}\right. \\
+H D D_{12} r^{2} x+H D D_{22} r^{2} y-2 H D_{1} D_{2} r^{2} x-2 H D_{2}^{2} r^{2} y \\
+D^{2} H_{1} x y-2 D^{2} H_{2} x^{2}-D^{2} H_{2} y^{2}-D^{2} H_{12} r^{2} x \\
-D^{2} H_{22} r^{2} y+D D_{1} H_{2} r^{2} x+D D_{2} H_{1} r^{2} x+2 D D_{2} H_{2} r^{2} y, \\
\Omega^{02}=-\frac{1}{16 \pi D^{3} r^{3}}\left(H D^{2} x-H D D_{2} x y+2 H D D_{1} y^{2}+H D D_{1} x^{2}\right. \\
+H D D_{12} r^{2} y+H D D_{11} r^{2} x-2 H D_{1} D_{2} r^{2} y-2 H D_{1}^{2} r^{2} x \\
+D^{2} H_{2} x y-2 D^{2} H_{1} y^{2}-D^{2} H_{1} x^{2}-D^{2} H_{12} r^{2} y \\
-D^{2} H_{11} r^{2} x+D D_{2} H_{1} r^{2} y+D D_{1} H_{2} r^{2} y+2 D D_{1} H_{1} r^{2} x, \\
\Omega^{03}=\Omega^{30}=0 .
\end{array}
$$

We see that for $H=e^{a r}$ and $D=e^{a r} / \sqrt{2}$, Eqs.(39)-(42) yield

$$
\begin{gathered}
\Omega^{00}=\frac{(1-a r)}{16 \sqrt{2} \pi r^{3}}\left(e^{a r}+2 r^{2} e^{-a r}\right), \\
\Omega^{01}=\frac{y}{8 \sqrt{2} \pi r^{3}}, \\
\Omega^{02}=-\frac{x}{8 \sqrt{2} \pi r^{3}},
\end{gathered}
$$




$$
\Omega^{03}=\Omega^{30}=0 .
$$

These turn out to be the energy and momentum density components for Gödel spacetime given by Sharif [19].

\section{Discussion}

It has been remained a controversial problem whether energy and momentum are localizable or not. Misner et al [30] were the points of view that energy can only be localized for spherical systems. Cooperstock and Sarracino [31] argued that if energy can be localized in spherical systems then it can be localized in any spacetimes. Bondi [32] supported the point of view that a non-localizable form of energy cannot be allowed in GR and hence its localization can be found in principle. The energy-momentum complexes are non-tensorial under general coordinate transformations and hence are restricted to Cartesian coordinates only. Virbhadra and others have shown [14]-[19] that these energy-momentum complexes can provide meaningful results.

In this paper, we have evaluated the energy and momentum density components for Gödel-type metrics by using prescriptions of Einstein and Papapetrou. It can be seen that the energy and momentum densities turn out to be finite and well defined in both the prescriptions. These provide general results in terms of $H$ and $D$ which can furnish interesting results for special values of $H$ and $D$. It follows from Eqs.(19)-(24) and (39)-(42) that the two results obtained by using the Einstein and Papapetrou energy-momentum complex differ in general for Gödel-type metrics. This should be considered important why the two results are different. It is to be noted from Eqs.(25)(28) and (43)-(46) that both the results reduce to the known energy and momentum densities of a Gödel metric as given in [19].

There are spacetimes [14], [19], [33] for which the two or more energy momentum complexes do not give the same result. We have exposed another model for which the two energy-momentum complexes do not provide the consistent result. This is another example which indicate that the idea of localization does not follow along the lines of pseudo-tensorial construction but instead it follows from the energy-momentum tensor itself. 


\section{Acknowledgment}

The author would like to thank Higher Education Commission (HEC), Pakistan for providing postdoctoral fellowship at University of Aberdeen, UK. I am also grateful for the useful comments made by the anonymous referee.

\section{References}

[1] Trautman, A.: Gravitation: An Introduction to Current Research ed. Witten, L. (Wiley, New York, 1962)169.

[2] Landau, L.D. and Lifshitz, E.M.: The Classical Theory of Fields (AddisonWesley Press, Reading, MA, 1962)2nd ed.

[3] Tolman, R.C.: Relativity, Thermodynamics and Cosmology (Oxford Univ. Press, 1934)227.

[4] Papapetrou, A.: Proc. R. Irish. Acad. A52(1948)11.

[5] Bergmann, P.G. and Thompson, R.: Phys. Rev. 89(1953)400.

[6] Weinberg, S.: Gravitation and Cosmology (Wiley, New York, 1972).

[7] Möller, C.: Ann. Phys. (NY) 4(1958)347.

[8] Möller, C.: Ann. Phys. (NY) 12(1961)118.

[9] Komar, A.: Phys. Rev. 113(1959)934.

[10] Penrose, R.: Proc. Roy. Soc. London A381(1982)53.

[11] Kovacs, D.: Gen. Rel. and Gravit. 17(1985)927;

Novotny, J.: Gen. Rel. and Gravit. 19(1987)1043;

[12] Bergqvist, G.: Class. Quantum Gravit. 9(1992)1753.

[13] Bernstein, D.H. and Tod, K.P.: Phys. Rev. D49(1994)2808. 
[14] Virbhadra, K.S.: Phys. Rev. D60(1999)104041.

[15] Virbhadra, K.S.: Phys. Rev. D41(1990)1081; D42(1990)1066; D42(1990)2919; and references therein.

[16] Xulu, S.S.: Int. J. Mod. Phys. A15(2000)2979; Mod. Phys. Lett. A15(2000)1511 and references therein.

[17] Yang, I.C. and Radinschi, I.: Mod. Phys. Lett. A17(2002)1159.

[18] Sharif, M.: Int. J. of Mod. Phys. A17(2002)1175.

[19] Sharif, M.: Int. J. of Mod. Phys. A18(2003); Erratum A(2003).

[20] Lessner, G.: Gen. Rel. Grav. 28(1996)527.

[21] Cooperstock, F.I.: in Topics on Quantum Gravity and Beyond, Essays in honour of Witten, L. on his retirement, ed. Mansouri, F. and Scanio, J.J. (World Scientific, Singapore, 1993); Mod. Phys. Lett. A14(1999)1531; Annals of Phys. 282(2000)115;

Cooperstock, F.I. and Tieu, S.: Found. Phys. 33(2003)1033.

[22] Xulu, S.S.: Mod. Phys. Lett. A15(2000)1511; Astrophys. and Space Science 283(2003)23.

[23] Bringley, T.: Mod. Phys. Lett. A17(2002)157.

[24] Som, M.M. and Raychaudhuri, A.K.: Proc. R. Soc. A304(1968)81;

Banerjee A. and Banerji, S.: J. Phys. A: Gen. Phys. 1(1968)188.

[25] Raychaudhuri, A.K. and Thakurta, S.N.: Phys. Rev. D22(1980)802.

[26] Reboucas, M.J. and Tiomno, J.: Phys. Rev. D28(1985)1251.

[27] Reboucas, M.J. and Aman, J.E.: J. Math. Phys. 28(1987)888.

[28] Reboucas, M.J. and Teixeira, A.: J. Math. Phys. 33(1992)2885.

[29] Reboucas, M.J. and Aman, J.E.: J. Math. Phys. 40(1999)4011.

[30] Misner, C.W., Thorne, K.S. and Wheeler, J.A.: Gravitation (W.H. Freeman, New York, 1973)603. 
[31] Cooperstock, F.I. and Sarracino, R.S.: J. Phys. A.: Math. Gen. 11(1978)877.

[32] Bondi, H.: Proceedings of the Royal Society of London A427(1990)249.

[33] Yang, I.C. and Radinschi, I.: gr-qc/0309130. 